\title{
Determining the biomechanical changes in lumbar spine among patients with injuries around the knee joint: a systematic review protocol
}

\author{
Sumit Raghav*, Anshika Singh \\ Subharti College of Physiotherapy, Swami Vivekakanand Subharti University, Meerut, U.P, India \\ Received: 08 June 2020 \\ Revised: 13 July 2020 \\ Accepted: 15 July 2020 \\ *Correspondence: \\ Dr. Sumit Raghav, \\ E-mail: drsumitraghav@gmail.com \\ Copyright: ( $)$ the author(s), publisher and licensee Medip Academy. This is an open-access article distributed under \\ the terms of the Creative Commons Attribution Non-Commercial License, which permits unrestricted non-commercial \\ use, distribution, and reproduction in any medium, provided the original work is properly cited.
}

\begin{abstract}
The knee is one of the body's more complicated joints and is susceptible to various injuries. A closed kinetic relationship exists between the lumbar spine and knee joint and any dysfunction in knee or lumbar region may result in compensation, joint dysfunction and pain in either one or both the regions. Therefore, the above stated reason highlights the need of analyzing the spine biomechanically. So, that a new physiotherapeutic assessment and treatment approach can be developed in future to prevent permanent adaptive changes in the posture in patients with knee injuries. This systematic review protocol has been conducted by two independent reviewers who searched the articles using electronic search for publications in several databases: Google Scholar, Index Copernicus, JSTOR, PubMed/Medline, ScienceDirect, Scopus and Web of Science. After applying the selection criteria, study papers published between the years 2001 to 2019 have been selected. Studies of human participants of any age having injuries around knee joint have been eligible, and there was no restriction on type of injuries. All the study papers have been analyzed using modified Downs and Black scale and scores have been awarded for the items selected on a 27 point scale. This review has collected secondary data only; hence no ethical approval was required. The results of this review protocol will provide evidence regarding changes in lumbar spine in patients with injuries around knee and this information will be useful in planning for rehabilitation in knee and around knee injury.
\end{abstract}

Keywords: Lumbar spine biomechanics, Knee injuries, Knee joint

\section{INTRODUCTION}

Musculoskeletal injuries are the most common form of sports and other physical activity injuries, representing 80 percent of injuries. ${ }^{1}$ Knee joint, which is the most often injured part of the body and knee joint fractures, constitutes a large number of injuries in both professional and recreational sports. $^{2}$ Physical activity plays an significant role in improving health and well-being for people of all ages and has been responsible for lowering health risks and the index of body mass, increasing physical skills and social interaction. ${ }^{3}$ But physical activity often carries a risk of injury, which can threaten an individual's physical, psychological, social, mental, and economic well-being. Participation in sports and other physical activities among young adults is considered a significant risk factor for knee injury. ${ }^{4}$

Knee joint is the most frequently injured joint in teenage athletes in the United States, with an estimated 2.5 million injuries each year. This form of injury typically involves substantial surgery and lengthy recovery. ${ }^{5} \mathrm{~A}$ knee injury can affect physical activity and physical ability temporarily and to a certain degree permanently. ${ }^{6}$ However, while knee injuries may have an economic impact on injured individuals, they also place a burden on the health care system. ${ }^{7}$ Human body is a collection of multiple segments and all segments of the body function 
together in a closed cinematic chain, and if some change occurs in the structure of one segment then it is directly or indirectly responsible for producing compensatory changes in the adjacent ones sections and junctions. ${ }^{8}$ Reduction of the range of motion of the knee joint contributes to increased movement of the pelvis and the shift in kinematic activity affects the strength and stability of the lumbar spine and may cause pain and weakness in the lumbar region of the spine. ${ }^{9}$

Through a closed kinetic chain the body works together and any change through movement at one joint affects activity at other joints. Ground reaction forces operate through feet, knees, and legs linkages through the spine and pelvis. Distortions in the spinal and pelvic regions may be due to altered knee joint biomechanics. ${ }^{10}$ The association between low back pain or conditions and knee pain can be explained across different factors. There is a closed kinetic relationship between the lumbar spine and the knee joint, and any dysfunction in the knee or lumbar region can cause compensation, joint dysfunction and pain in either region or both. ${ }^{11}$

Knowing this relationship between the biomechanical variations in the knee joint and its impacts on the spinal position and loading can enhance the efficacy of individual case management. Directing physical therapists' efforts to analyze and examine the patient's whole body posture and not only concentrating their efforts on the symptomatic area is only important, as improvements in the knee posture may have long-term effects in both the spinal and pelvic regions. ${ }^{12}$ Directing physical therapists' attention to determine and analyze the patient's entire body posture and not merely concentrating their attention on the symptomatic area is important primarily because improvements in knee posture may have long-term effects in both the spinal and pelvic regions. Hence, the explanation given above highlights the need for biomechanical analysis of the spine. So that a new strategy may be implemented in the future to avoid irreversible functional posture changes with regard to improvements in the lumbar spine in patients with knee injuries. This analysis will help to create a relationship between knee injuries and biomechanical changes in loading the spinal cord.

\section{Objectives}

The primary objective of this review was to evaluate systematically the biomechanical changes in lumbar spine among patients with injuries around the knee.

\section{Key review question}

What is the impact of injuries around the knee on lumbar spine biomechanics?

$\mathrm{P}=$ adult patients with injuries around knee; $\mathrm{I}=$ no intervention; $\mathrm{C}=$ any comparator; $\mathrm{O}=$ any measure of outcome related to assessment of functions and load on lumbar spine; $S=$ any kind of setting in any area/country.

\section{Secondary review questions}

What precautions and contraindications need to be taken into account while evaluating the changes in lumbar spine biomechanics in patients with injuries around knee?

The articles selected from the search findings for the key review question will be reviewed further to answer the secondary review question. Selected articles will be investigated for additional information.

What challenges are faced by the therapist while evaluating the changes in lumbar spine biomechanics in patients with injuries around knee?

\section{METHODS}

\section{Study design}

This was a review protocol based on principles of the preferred reporting items for systematic reviews and meta-analysis protocols (PRISMA-P).

\section{Eligibility criteria}

Type of study

All cross-sectional studies evaluating the impact of knee injury and injury around the knee on changes of lumbar spine have been included in this systematic review, including feasibility studies, pilot studies, experimental studies and quasi-experimental studies. RCTs, review articles, editorials, case studies, qualitative studies, animal studies and study protocols have been excluded.

\section{Participants}

Studies have been involved only human participants of any age with injuries around knee. There was no restriction on unilateral or bilateral involvement of knee. Both the genders i.e. males and females have been included. Studies involving animals as participants have been excluded from the review. Studies with patients suffering with other acute/chronic serious illness have been excluded from the study. Studies involving mentally challenged or psychosocially unfit patients have also been excluded in this review protocol.

\section{Interventions}

Studies without interventions have been included in this review. Any comparator has been included.

\section{Comparisons or control}

Studies addressing impact of knee injury and injuries around the knee on biomechanical change in lumbar spine 


\section{Outcome measures}

Outcomes measures for evaluation of biomechanics to monitor the motions of lumbar spine, forces acting on lumbar spine with validated tools for functional assessment and spinal load assessment should be used at least once in the studies have been included.

\section{Language}

We have only considered full text articles published in English.

\section{Search strategy for identification of relevant studies}

A comprehensive search has been conducted at the central library of Swami Vivekanand Subharti University (SVSU) and library of Subharti College of Physiotherapy, Meerut during the period of 6-9 months. All bibliographic databases of published research papers which were easily accessible have been assessed. All databases included have been searched and all the papers published till 2019 have been included. The electronic database such as Google Scholar, Index Copernicus, JSTOR, PubMed/ Medline, ScienceDirect, Scopus and Web of Science have been included for search the articles.

\section{Study records}

All the searched results have been merged using reference management software 'Mendeley'. The result of electronic searches has also been saved to the researcher's account on PubMed. The principal investigator/researcher has created a shared folder on 'Google drive' to encourage and facilitate collaboration among reviewers and to make it accessible by all the researchers. The physical backup has been maintained by keeping the printed copies of summaries of all the screened articles.

\section{Data extraction}

Data from included studies has been extracted using a standardized data extraction form. Two reviewers, both physiotherapists, have searched the databases independently and screen the titles and abstracts for eligibility. The searched titles and abstracts have been examined carefully and irrelevant reports will be removed. The full text of selected potentially relevant articles has been obtained; multiple articles of the same study on different database will be linked to minimize the duplication. Both the reviewers have thoroughly assessed full text articles to check their compliance in accordance with the inclusion and exclusion criteria. The data analysis/ synthesis of the articles meeting the eligibility criteria have been done by the one reviewer. The reviewer will also search the references for articles manually to include in data extraction.

\section{Risk of bias}

To assess the risk of bias the Cochrane collaboration tool has been used. To assess the quality of literature modified Downs and Black 27 point scale was used.

\section{Quality of evidence}

A narrative synthesis of the selected studies from the search findings has been provided due to the likely heterogeneity of the outcome measures. Patient's population and outcome measures were described in a narrative summary. Information on adherence to the protocol, resources used, compliances monitoring and expenditure was extracted from the selected studies, if available.

\section{DISCUSSION}

In our knowledge, this article is the first review protocol on related to evaluate the biomechanical changes in lumbar spine among patients with injuries around the knee. Previous reviews have been investigated. In this view, a study was conducted at Chiba University, Japan by Murata et al, in 2003, to find out the relationship between lumbar lordosis and extension of the knee. A total of 366 patients were included in the study and all the patients underwent radiological examination of the lumbar spine in standing position. Physical examination using a goniometer was done to assess the knee and body angles. In patients with lumbar lordosis of $30^{\circ}$ or less, limitation of extension of the knee joint was significantly greater. In patients with limitation of extension of the knee joint of $5^{\circ}$ or more, lumbar lordosis was significantly reduced. The study indicated that the degenerative changes in the knee cause the lumbar spine symptom. ${ }^{13}$

A study was conducted by Alfeky, Draz and Elsayed, in 2016, in isokinetic lab at Cairo University to measure lumbar proprioception in patients with chronic unilateral knee osteoarthritis (grade II). A total of 60 individuals were evaluated and assessed for lumbar proprioceptive accuracy using Biodex system 3, Pro Multi-joint system isokinetic dynamometer. The study provided evidence on the effects of knee osteoarthritis on lumbar proprioception and directed the attention of the physiotherapists to assess and evaluate the complete body posture of the patient and to not to just focus their entire attention on the symptomatic area is important only, as changes in the posture of the knee can produce long-term effects both in spinal and pelvic regions. ${ }^{14}$

Another study was conducted by Kahrizi et al, in 2007, to evaluate spinal internal loads and curvature of the lumbar spine under holding static load at different trunk and knee positions. The purpose of this study was to determine the effects of the external load on curvature of the lumbar spine and spinal loading, to understand the risk of injuries to the back in static holding tasks. Ten healthy male 
individuals with mean age of $23.2 \pm 1.3$ years were included in this study. The results have shown that interaction between load and trunk position was significant. The value of shear force was greater in tasks performed with combination of trunk flexion at $30^{\circ}$ and straight knee due to increase tilting of pelvis anteriorly. ${ }^{15}$

In continuation with supporting previous literatures, a study was designed by Yanagisawa et al, in 2015, as an investigative method to assess the association between the knee joint and spinal alignment, using the Spinal Mouse. The Spinal Mouse was used to measure range of motion and alignment of the spine in sagittal plane. The study population included 264 participants with a mean age of $71.1 \pm 6.8$ years. A questionnaire involving symptoms in the knees joint was administered, and physical examinations to assess the range of motion of knee were done. To conduct a comparative study, the patients were divided into two groups, one group with knee flexion contracture and another group without knee flexion contracture. The study concluded that lumbar lordosis angle, sacral inclination angle, spinal range of motion, lumbar lordosis angle and trunk angle of inclination decreased significantly in the flexion contracture group. ${ }^{16}$ This review will provide an answer to the question on the effect of injuries around knee on biomechanics of lumbar spine. Information related to the safety measures that have been taken to prevent adverse events during the biomechanical analysis of lumbar spine will be included.

\section{CONCLUSION}

In this paper, a novel biomechanical evaluation for spinal loading among the patients with injuries around the knee has been proposed. The evaluation was based on biomechanical perspective regarding the function and loading parameters of the lumber spine in patients with injuries around the knee joint. Evaluation format of function and loading of the lumber spine has been formulated for rule out changes in lumbar spine due to alteration of walking pattern among the patients with injuries around the knee. Furthermore, this review article will be very helpful to carry out the systematic review on the evaluation of biomechanical changes of the lumbar spine among the patients with injuries around the knee joint.

Funding: No funding sources Conflict of interest: None declared

Ethical approval: Not required

\section{REFERENCES}

1. Nicolini AP, Matsuda MM, Filho JS, Cohen M. Common injuries in athletes' knee: experience of a specialized center. Acta Ortop Bras. 2014;22(3):127-31.

2. Murphy DF, Connolly DAJ, Beynnon BD. Risk factors for lower extremity injury: a review of the literature. Br J Sports Med. 2003;37:13-29.
3. McPhee JS, French DP. Nazroo JJ, Pendleton N, Degens H. Physical activity in older age: perspectives for healthy ageing and frailty. Biogerontology. 2016;17:567-80.

4. Malm C, Jakobsson J, Isaksson A. Physical Activity and Sports-Real Health Benefits: A Review with Insight into the Public Health of Sweden. Sports. 2019;7:127.

5. Brett, E, Gage et. al. Epidemiology of 6.6 Million Knee Injuries Presenting to United States Emergency Departments From 1999 Through 2008. Acad Emerg Med. 2012;19(4):378-85.

6. Jayabalan P, Ihm J. Rehabilitation Strategies for the Athletic Individual with Early Knee Osteoarthritis. Curr Sports Med Rep. 2016;15(3):177-83.

7. Vleeming A, Schuenke MD, Masi AT, Carreiro JE, Danneels L, Willard FH. The sacroiliac joint: an overview of its anatomy, function and potential clinical implications. J Anat. 2012;221:537-67.

8. Proske U, Gandevia SC. The proprioceptive senses: their roles in signaling body shape, body position and movement, and muscle force. Physiol Rev. 2012;92:1651-97.

9. Myer GD, Kushner AM, Brent JL, Schoenfeld BJ, Lloyd JHRS, Vermeil A, et al. The back squat: A proposed assessment of functional deficits and technical factors that limit performance. Strength Cond J. 2014;36(6):4-27.

10. Lu TW, Chang CF. Biomechanics of human movement and its clinical applications. Kaohsiung $\mathbf{J}$ Med Sci. 2012;28:S13-S25.

11. Cook G, Burton L, Hoogenboom BJ, Voight M. Functional movement screening: the use of fundamental movements as an assessment of function- part 1. Int $\mathbf{J}$ Sports Phys Ther. 2014;9(3):396-409.

12. Delitto A, George SZ, Dillen LV, Whitman JM, Sowa GA. Low Back Pain. J Orthop Sports Phys Ther. 2012;42(4):1-57.

13. Murata Y, Takahashi K, Yamagata M, Hanaoka E, Moriya H. The Knee-Spine Syndrome. Association Between Lumbar Lordosis and Extension of the Knee. J Bone Joint Surg Br. 2003;85(1):95-9.

14. Alfeky FM, Draz AH, Elsayed WH. The Effect of Knee Osteoarthritis on Lumbar Proprioception. Int J PharmTech Res. 2016,9(4):80-91.

15. Kahrizi S, Parnianpour M, Firoozabadi SM, Kasemnejad A, Karimi E. Evaluation of Spinal Internal Loads and Lumbar Curvature Under Holding Static Load at Different Trunk and Knee Positions. Pak J Biol Sci. 2007;10(7):1036-43.

16. Yanagisawa S, Sato N, Shimiz M, Saito K, Yamamoto A, Takagishi K. Relation among the knee, sagittal spinal alignment, and the spinal range of motion: Investigation in local medical check-ups using the Spinal Mouse. Asia Pac J Sports Med Arthrosc Rehabil Technol. 2015;2:68-71.

Cite this article as: Raghav S, Singh A. Determining the biomechanical changes in lumbar spine among patients with injuries around the knee joint: a systematic review protocol. Int J Community Med Public Health 2020;7:3251-4. 